Nouvelles perspectives en sciences sociales

Revue internationale de systémique complexe et d'études relationnelles

\title{
Des dialectiques relatives aux médias et à la culture dans la francophonie canadienne
}

\section{Simon Laflamme}

Volume 8, numéro 2, mai 2013

Sur le thème de représentations identitaires et expressions culturelles de la francophonie canadienne à travers ses pratiques artistiques et médiatiques

URI : https://id.erudit.org/iderudit/1016469ar

DOI : https://doi.org/10.7202/1016469ar

Aller au sommaire du numéro

\section{Éditeur(s)}

Prise de parole

\section{ISSN}

1712-8307 (imprimé)

1918-7475 (numérique)

Découvrir la revue

\section{Citer cet article}

Laflamme, S. (2013). Des dialectiques relatives aux médias et à la culture dans la francophonie canadienne. Nouvelles perspectives en sciences sociales, 8(2), 29-50. https://doi.org/10.7202/1016469ar

\section{Résumé de l'article}

La culture, dans son sens sociologique, est le résultat d'une dialectique toujours en cours entre des symboliques et des ensembles d'individus, d'une part, et entre des individus entre eux et des collectifs entre eux, d'autre part; elle est information et communication. Dans les sociétés hautement définies par les moyens de communication de masse, cette dialectique non seulement est-elle mouvement continu, mais elle est encore pluralité. Cette pluralité remet en cause la possibilité même de l'identité sociale. S'opère ainsi une autre dialectique, celle-ci entre homogénéisation et différenciation. Les médias constituent l'un des facteurs les plus déterminants des symboliques, et donc des identités. Les messages qu'ils émettent sont soumis aux diverses contraintes de la concurrence, notamment à celles de l'économie et de la démographie, mais aussi à celles de la représentation et de la dialectique entre les producteurs de message et leurs destinataires. Une collectivité, dans la contemporanéité, ne peut se reproduire que dans la mesure où elle évolue dans ce complexe. C'est ce dont nous tenterons de rendre compte en mettant l'accent sur la francophonie canadienne. 


\title{
Des dialectiques relatives aux médias et à la culture dans la francophonie canadienne
}

\author{
Simon LAfLAmme \\ Université Laurentienne, Sudbury
}

\section{Introduction}

Dar son titre, le numéro thématique désigne un univers pluridimensionnel. Rappelons ce titre : «Représentations identitaires et expressions culturelles de la francophonie canadienne à travers ses pratiques artistiques et médiatiques ». Il couvre ainsi divers registres : ceux

i. des représentations identitaires,

ii. des expressions culturelles,

iii. de la francophonie canadienne,

iv. des pratiques artistiques,

v. et des pratiques médiatiques.

Chacun de ces registres a ses repères théoriques et, encore plus nombreuses, ses manifestations empiriques. Il peut donner lieu, en lui-même, à un vaste inventaire de travaux d'interprétation, de modélisation et d'observation, de même qu'il ouvre sur un large éventail de recherches possibles. Et s'il en est ainsi, c'est que des communautés de savants trouvent en chacun d'eux des problématiques importantes aux plans aussi bien scientifique que 
social. Ces registres peuvent être combinés deux à deux. Le spécialiste examinera alors les relations entre, par exemple, les pratiques médiatiques et les représentations identitaires, ou entre les expressions culturelles et la francophonie canadienne, ou entre les pratiques artistiques et les pratiques médiatiques. Ces combinaisons multiplient toutes la liste des travaux effectués ou virtuels puisqu'elles réunissent deux ensembles distincts et qu'elles ont toutes, de surcroît, une intersection. Ces registres peuvent encore être réunis à plus de deux. Le champ des recherches s'étend alors davantage, mais beaucoup plus en ce qu'il associe des travaux spécifiques aux divers registres et à plusieurs intersections de deux d'entre eux qu'en ce qu'il permet de recenser des études à ces niveaux de combinaison. Il est peu facile, pour les scientifiques, d'opérationnaliser à l'intersection de trois de ces champs, ce qui raréfie la réalisation de travaux à cette échelle. Et la difficulté s'accroît si l'ensemble comprend quatre de ces registres. Or, les directeurs de ce numéro thématique ont rassemblé les cinq champs.

Pourquoi l'ont-ils fait? On pourrait le leur demander. Mais on peut croire qu'ils nous feraient alors des réponses comme les suivantes :

i. pour favoriser les liens entre les spécialistes de ces registres,

ii. pour décloisonner la recherche,

iii. pour tenter de repousser les frontières des travaux de recherche.

Ces trois réponses - qui sont en fait du même ordre - sont louables. Mais il y a plus - et les directeurs le signaleraient sans doute -; c'est que, empiriquement, ces cinq champs ne peuvent pas ne pas être interconnectés dès lors qu'ils ont pour questionnement la possibilité pour la francophonie canadienne de se reproduire. 


\section{2. Éléments de théorisation d'une vaste problématique}

Dans la postindustrialité, il n'y a pas de communauté linguistique sans expressions culturelles, sans pratiques artistiques, sans pratiques médiatiques, sans identité aucune. On pourrait ainsi être tenté de dessiner une figure étoilée (voir Figure 1).

Figure 1

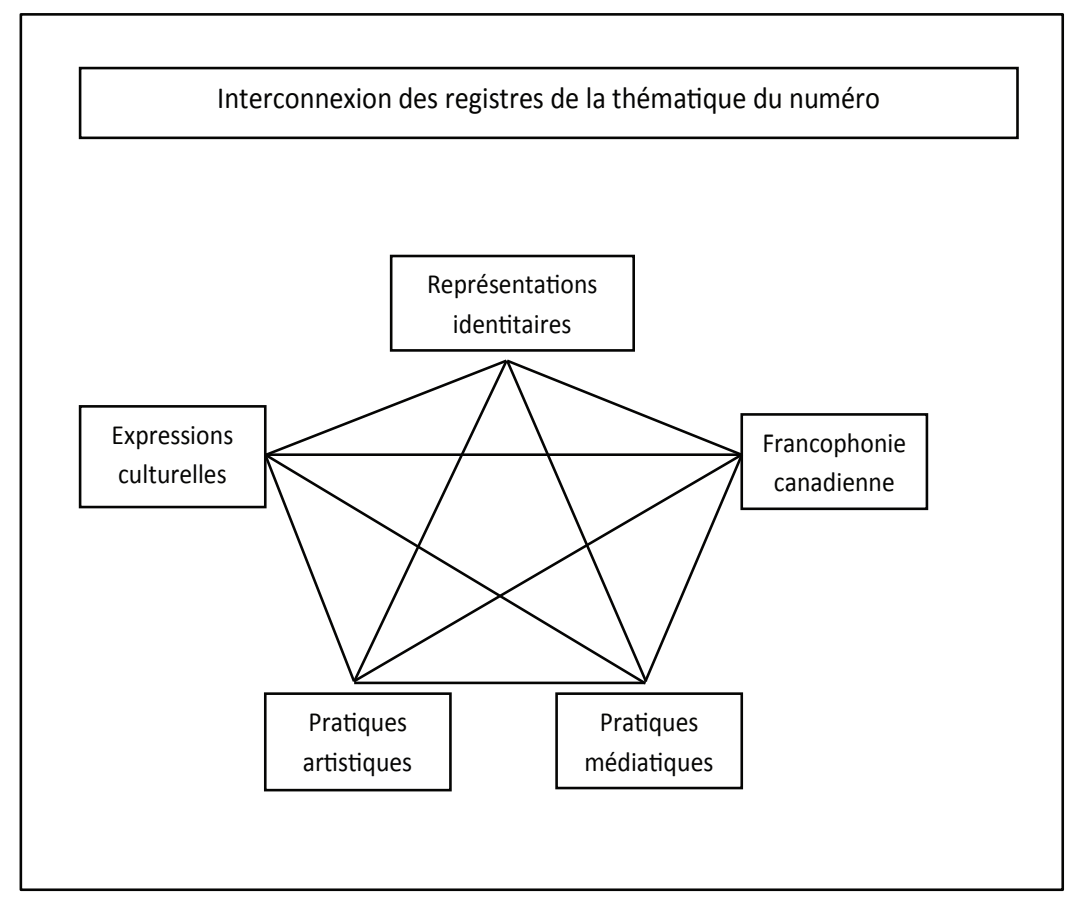

Certes, cette représentation est trop simple : tous ces registres ne sont pas du même ordre, ni les relations entre les éléments ne sont de même nature.

Il y a d'abord le caractère singulier ou pluriel de certains référents qui complique la compréhension de la structure. Le terme représentations identitaires, par exemple, peut renvoyer à des systèmes idéels relatifs à des individus, comme il peut désigner des collectifs. Entre ces deux réalités, il y a dialectique; mais chacune d'elles jouit d'une relative autonomie. L'identité, chez l'individu, si elle est toujours pénétrée de formes collectives, elle 
n'est jamais assimilable à ces formes et cette relative indépendance de l'individualité est décuplée par la complexification de la socialité, c'est-à-dire par l'accroissement des sources d'information et par la variété des interactions entre ces sources. La sphère collective des représentations identitaires, elle, n'est pas que la somme des imaginaires individués; elle possède une constitution en propre. Les expressions culturelles peuvent se rapporter à la production d'œuvres spécifiques; dans cette appellation, dans bien des cas, elles se distinguent peu des produits artistiques, mais leur univers se veut plus étendu. Elles peuvent aussi connoter le sens sociologique et rappeler l'espace symbolique qui anime une collectivité.

Les pratiques artistiques sont des faires humains qui reposent sur des compétences. Le terme est beaucoup moins polysémique que ne le sont ceux d'expressions culturelles et de représentations identitaires, quoiqu'il renvoie à une réalité multiforme.

Dans les représentations identitaires d'une communauté, il entre forcément des pratiques artistiques : des rythmes, des architectures, des danses, des chansons... Mais tout ce qui est représentation identitaire n'est pas pratique artistique ou produit de cette pratique. Les produits artistiques sont souvent des manifestations de représentations identitaires collectives; toutefois, leur autonomie par rapport à cette identité peut être énorme quand, par exemple, sont mobilisées des techniques qui débordent le cadre d'une collectivité ou lorsque les modes d'expression transcendent la communauté d'appartenance. Si les représentations identitaires interviennent dans les pratiques artistiques, celles-ci n'agissent pas de la même manière sur celles-là.

En fait une structure étoilée ne vaut que parce qu'il est vrai que ses composants sont interconnectés quand on réfléchit sur l'existence d'une communauté. L'intuition, d'ailleurs, construit une telle structure qu'on trouvera parfois dans les positions éditoriales ou dans les propos idéologiques. Mais l'ensemble théorique est moins égalitaire, moins symétrique (voir Figure 2). Qui dit francophonie canadienne, dit nécessairement représentations identitaires, mais la francophonie canadienne n'est pas 
que représentations identitaires. Il n'y a pas de communauté linguistique nationale sans représentations identitaires. Ces représentations impliquent une constitution du soi par rapport à d'autres communautés (une espèce d'autopoï̀se activée par le paradoxe de l'autre et du soi, pour parler comme Niklas Luhmann ${ }^{1}$ ). Les représentations identitaires sont largement des expressions culturelles, au sens sociologique, et elles interviennent dans ces expressions comme le font aussi d'autres facteurs dont elle est pénétrée et qui subissent son influence. Dans ces expressions culturelles, interviennent, entre autres, des pratiques artistiques. Dans les sociétés hautement définies par les moyens de communication de masse, l'identité au niveau de l'ensemble suppose l'existence de médias qui diffusent à grande échelle, qui traduisent les manifestations de l'identité commune, et donc qui, tout à la fois, agissent sur les expressions culturelles tout en étant agis par elles. Ces pratiques médiatiques, en outre, sont en relation avec d'autres aspects de la francophonie canadienne que ses expressions culturelles, avec d'autres communautés, de même qu'avec d'autres formes d'altérités, comme des technologies transculturelles, par exemple. Car une communauté n'est pas qu'expressions culturelles et n'évolue pas sans entretenir de liens avec d'autres.

$1 \quad$ Niklas Luhmann, Systèmes sociaux. Esquisse d'une théorie générale, traduit de l'allemand par Lukas K. Sosoe, Québec, Presses de l'Université Laval, [1984] 2010, 569 p. 
Figure 2

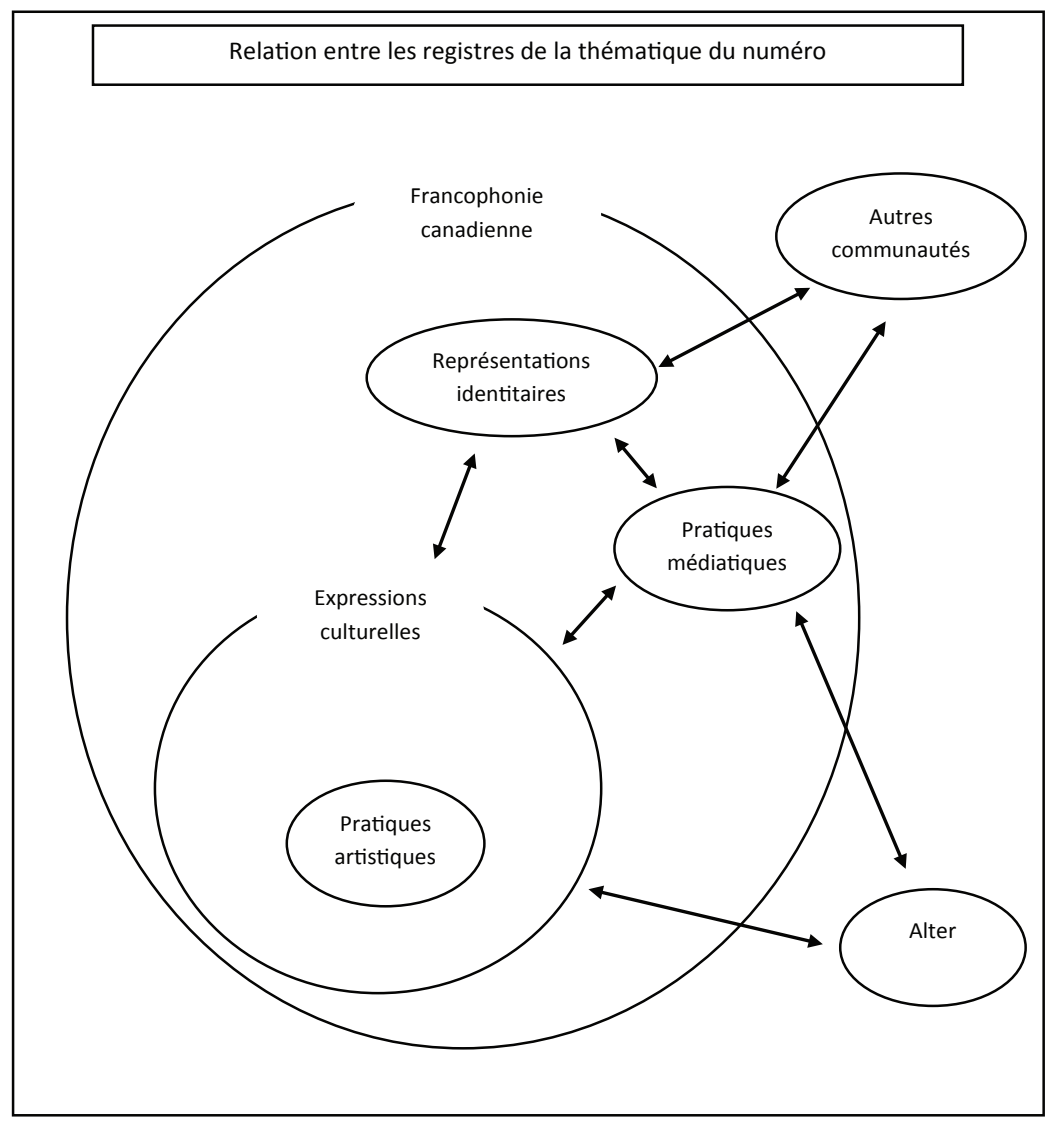

On a affaire à un ensemble théorisable intriqué, mais qui ne s'ouvre pas sur l'infini. Et l'altitude que donne l'abstraction permet de ne pas se perdre dans la multitude des éléments théoriques et empiriques; elle permet de ne pas empêcher une théorisation globale, sous prétexte du grand nombre des pratiques artistiques, de leurs relations entre elles, de leurs relations avec les autres aspects des expressions culturelles, du rapport entre toutes ces réalités et les autres spécificités de l'univers extraexpressions culturelles. 


\section{Symbolique et humanité}

La culture, au sens sociologique, c'est du symbole, du symbole partagé. C'est du symbole attaché à l'humanité de la socialité. Ce n'est pas que de la circulation d'information. C'est de la circulation d'information inscrite dans une dimension historique, c'est-à-dire qui participe d'un langage qui peut changer, d'un langage qui anime des locuteurs qui, eux-mêmes, modulent le langage, dynamique grâce à laquelle le langage et les locuteurs sont toujours en train de se construire. Grâce à cette dynamique, l'information humaine n'est pas que computation; elle est aussi cogitation (pour employer le vocabulaire d'Edgar Morin²). Grâce au langage, les humains agissent sur eux-mêmes et sont arrimés à une histoire en devenir (voir Figure 3).

Les symboles à travers lesquels et autour desquels se déploie la culture supposent du cogito; mais ils ne sont pas que rationnels. La culture est pénétrée de pensées, mais non pas dans un univers strictement conscient. La culture, c'est du conscient et de l'inconscient, c'est du rationnel fondu dans de l'émotif; c'est de l'émorationnel. C'est parce qu'elle n'est pas strictement raison que la culture anime les peuples. C'est parce qu'elle est raison qu'elle justifie les actions, individuelles et collectives, qu'elle permet que les sociétés s'organisent.

La culture, ce sont des symboles, construits entre le conscient et l'inconscient, entre la raison et l'émotion. C'est de la langue, mais ce n'est pas que de la langue. Si l'on peut partager des idées, on peut aussi partager des impressions, des peurs, des harmonies, des rythmes. L'humain imprègne presque tout de culture : l'alimentation, l'habitation, le rite. Aucun bien, par exemple, n'est strictement économique parce que la socialité les enveloppe tous de culture - il y a du symbolique dans une voiture, dans une diète, dans un manteau. Mais cette "culturation " ne se réduit ni à l'idéel, ni à la raison, ni à la langue. Certes, la langue constitue l'un des principaux véhicules de la culture; mais la culture circule aussi bien dans les phrases qu'au-delà et en deçà

2 Edgar Morin, La Méthode, tome 3, La Connaissance de la Connaissance. Anthropologie de la connaissance, Paris, Seuil, 1986. 
d'elles. Et c'est parce que tout n'est pas toujours dit dans les énoncés linguistiquement formulés, d'ailleurs, que la langue est toujours en devenir, comme quelque chose qui ouvre un espace à ce qui peut être dit et qui ne l'a pas encore été. C'est cet univers qu'exploiteront les artistes et les penseurs. Ces créateurs produiront des symboles dans lesquels les membres de la société se reconnaîtront. Cette découverte de soi sera possible parce que le créateur aura révélé quelque vérité que plusieurs portent déjà en eux ou parce qu'il aura trouvé quelque élément qui agit émorationnellement sur certains individus alors que les formes appartiennent à un autre éthos.

L'humanité de la socialité, c'est de l'histoire où des personnes, entre leur collectivité et leur individualité, agissent sur l'histoire qu'ils subissent dans et par un univers symbolique.

La culture, c'est du symbole partagé. C'est de l'information parce que c'est du symbole, c'est de la communication parce que c'est partagé. Il n'y a pas de culture en dehors d'un champ communicationnel. Mais toute information qui circule n'est pas réductible au lexique ou à la syntaxe. Le message contient souvent davantage que la formulation langagière qui lui sert de support. Et c'est parce que le langage porte en lui beaucoup plus des symboles que des sèmes. Le langage n'épuise pas la culture; il lui sert de support. La langue réunit des locuteurs autour des symboles auxquels elle donne une matière communicable.

La culture, c'est du symbole partagé. Ce sont donc des locuteurs en interaction. Ce sont des individus qui partagent une socialité et une historicité. Ce sont des agents communicants réunis autour de symboles. Ce sont des individus qui se font collectif parce qu'ils partagent des symboles et parce qu'ils peuvent se les communiquer dans un langage commun, la langue constituant l'un de ces principaux langages. La culture, ce sont des individus qui se reconnaissent dans la collectivité qu'ils forment et qui les fabrique. La culture, ce sont des humains qui font une collectivité qui les fait être comme individus, qui leur permet de se faire être socialement, de se socialiser, et donc de reconnaître leur être collectif en même temps que leur être propre. Le langage 
lui-même inscrit d'emblée l'humain dans la socialité parce qu'il est déjà collectivité. La culture, ce sont donc des rapports entre des individus, entre des individus et des collectifs. Dans la mesure où une collectivité en côtoie d'autres, son être spécifique ne peut se reproduire que si des symboliques permettent de la différencier. Il faut donc que, à travers les interactions interindividuelles qui la définissent, à travers les dialectiques du collectif et de ses membres, il soit possible de faire en sorte que la spécificité se produise ou se reproduise. Le collectif se donne alors une identité ou la pérennise.

Figure 3

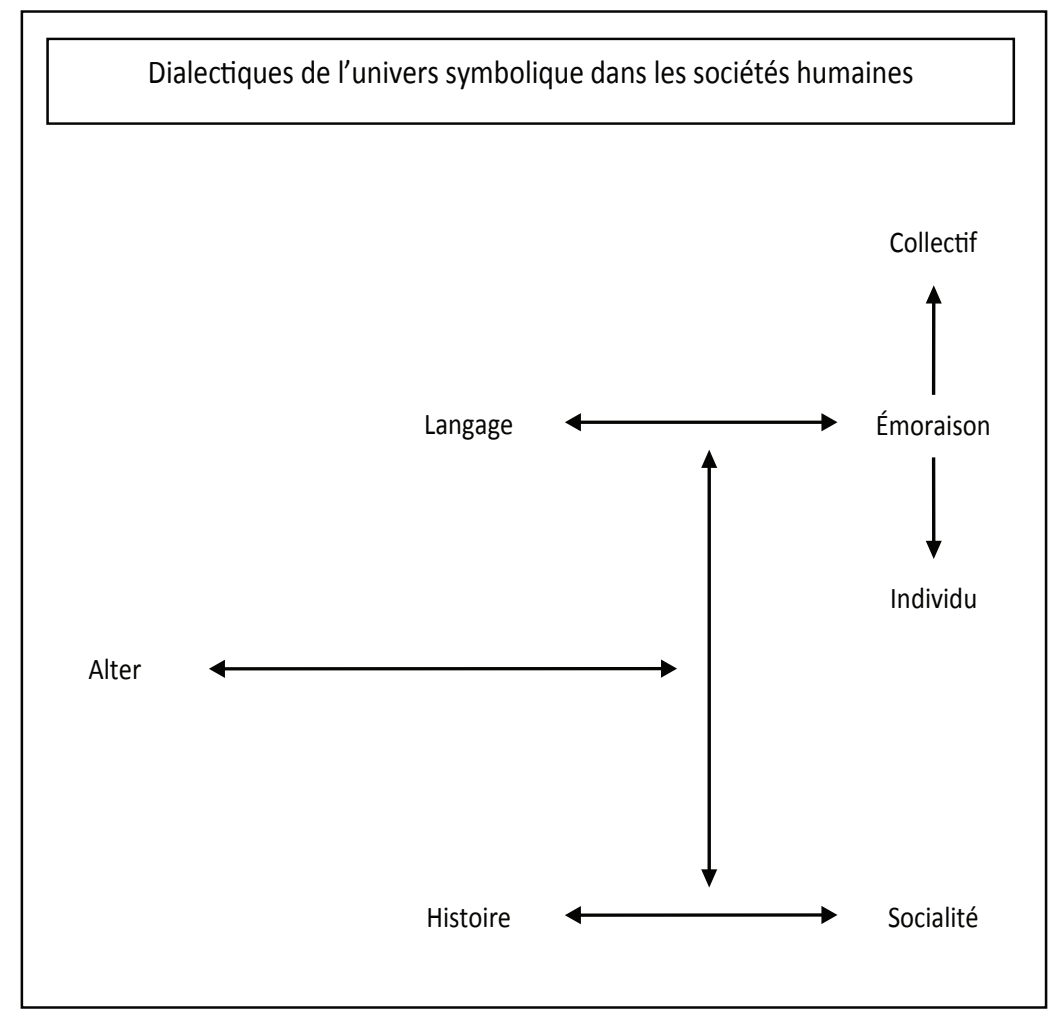




\section{Socialité et médias}

Quand la totalité des membres d'un collectif interagissent physiquement entre eux et que l'essentiel des informations qui circulent est le produit même de ces interactions, à travers les discussions, la danse, le spectacle, les œuvres plastiques, les tâches communes, alors les échanges in præsentia assurent le partage des symboles. Les médias ne sont alors pas nécessaires à la reproduction de la communauté. Dans la mesure où la population d'une société s'accroît, il devient moins probable pour les individus qui la composent de se côtoyer. Il ne peut alors y avoir de communauté que si l'ensemble des individus peut accéder à une information semblable. Le recours aux médias devient ainsi obligé. L'histoire accentue cette nécessité médiatique. En effet, puisque la société devient plus populeuse, elle change. Ce changement est modification de l'histoire; sa spécificité étant au moins partiellement faite de symboliques du passé, la société doit les faire circuler non seulement synchroniquement, mais aussi diachroniquement. Les médias favorisent cette transmission dans le temps.

L'histoire humaine est expansion des sociétés. Cette expansion est densification, ce dont les villes sont la manifestation la plus évidente; mais elle est aussi extension, ce dont témoignent les territoires nationaux. Ces développements ne peuvent avoir pour corollaire des communautés que si des médias de masse entrent en jeu. Il faut, en effet, que des personnes puissent s'exposer à des messages identiques par delà l'interaction des individus, à un même moment et en des lieux différents; il faut même que des individus puissent communiquer entre eux simultanément dans la non-unité de lieu. Les médias de masse, parce qu'ils s'adressent à plusieurs individus simultanément (radio, télévision ou Internet) ou parce qu'un même message peut être transmis à de grands ensembles de destinataires (imprimé, radio, télévision, Internet), mais aussi parce qu'ils rendent massivement possibles des échanges interindividuels (le téléphone, Internet), sont au fondement du développement sociétal. 
Mais le rôle des médias n'est pas que la diffusion. Les médias ne font pas que transmettre des messages qui proviennent de l'extérieur d'eux-mêmes. Ils peuvent faire cela; mais ils sont aussi des lieux de production de messages. Ils font souvent corps avec les messages qu'ils transmettent. Ce n'est pas que le message est le médium, comme le déclaraient Marshall McLuhan et Quentin Fiore $^{3}$; c'est que les médias peuvent modeler les messages qu'ils émettent. Les médias constituent des dispositifs de production de messages. Mais tout ce qui transite par les médias n'est pas purement médiatique. Il n'y a plus de communauté nationale sans un rapport aux médias de masse, mais tout ce qui est société n'est pas médias. Tout ce qui est message n'est pas médias. Et les médias peuvent tout aussi bien servir à la fabrication des messages qu'ils peuvent les relayer, même si ce relai impose quelque altération. Les médias font corps avec les sociétés contemporaines, mais cela ne veut pas dire qu'ils comprennent la totalité de ce qui peut être dit socialement. Un parti politique, dans la postmodernité, n'accédera pas au gouvernement s'il n'entretient pas de rapport avec les médias de masse; mais le message de ses militants n'est pas radicalement différent selon qu'ils l'expriment à la télévision ou qu'ils le communiquent lors d'une assemblée de comté.

Une communauté, avec la modernité, et de surcroît avec la postmodernité, ne peut assurer son existence que si elle dispose de médias, par le fait qu'elle ait pour corollaire l'étendue et la densité. Il lui faut produire des messages pour elle et se les communiquer en vertu du nombre de ses citoyens et du rapport qu'ont ces citoyens à un territoire donné. Si tel n'est pas le cas, elle ne peut se faire être à travers des symboliques partagées; elle ne peut donc se faire être, point. Si une communauté étendue ne dispose pas de médias, elle est à la merci des messages des autres - les informations qui lui sont transmises massivement n’ont été choisies ou créées ni pour elle ni par elle -; elle ne peut assurer, par ailleurs, les interactions interindividuelles en dépit

$3 \quad$ Marshall McLuhan et Quentin Fiore, The Medium is the Message: an Inventory of Effects, New York, Bantam Books et Random House, 1967. 
de la non-unité de lieu : sans téléphone, sans Internet, sans réseaux sociaux, il n'est pas aisé d'échanger avec des proches qui sont à des centaines de kilomètres. La possibilité pour une communauté donnée, en outre, de créer et de diffuser pour elle des messages de masse lui permet de se faire être aux yeux des autres communautés, ce qui, souvent, renforce l'être collectif intérieur.

\section{Médias et minorités}

Les communautés contemporaines ont besoin de médias pour se faire être. Les médias ne leur suffisent pas, certes. Il leur faut bénéficier d'un ensemble important d'institutions : d'écoles, de centres récréatifs, de milieux artistiques, d'organismes de gens d'affaires, d'institutions financières... Il ne s'agit donc pas d'une condition suffisante; il s'agit d'une condition nécessaire.

Mais les communautés, dans la contemporanéité, c'est aussi de la diversité. Et les médias, c'est de la concurrence. Cela complexifie l'identification collective et la reconnaissance des expressions culturelles.

Une communauté cohabite souvent avec d'autres. Généralement, elle partage avec ces autres certains traits. La reproduction de ces caractéristiques peut donc être assurée par les médias qui ont pour destinataire la communauté globale. Mais, dans la mesure de sa spécificité, il lui faut des médias en propre. La langue, par exemple, distingue grandement une population. En fait, elle est l'un des plus grands facteurs de différenciation culturelle, avec la religion. Dans la mesure où elle particularise, si les messages qui s'adressent à l'ensemble de ses membres lui viennent dans une autre langue, elle ne peut reproduire collectivement cette dimension de son être (voir Figure 4).

La place qu'occupe une langue dans une société donnée, c'est déjà du symbolique, c'est déjà de la culture.

Tout ce qui est culturel n'est pas langue; et c'est pourquoi deux langues différentes peuvent avoir des traits culturels communs. Mais il n'y a pas de culture sans langue. Ainsi, la langue module la culture. 
Le fait d'appartenir à une minorité ou à une majorité linguistique correspond à une position sociale qui imprègne la culture. Mais on n'a pas ici affaire à une corrélation de cause à effet qui voudrait que minoritaire corresponde à dévalorisation de soi et majoritaire à l'inverse. Ces dynamiques sont très compliquées. Il est des peuples minoritaires valorisés par eux-mêmes et par les autres de même que des peuples majoritaires dévalorisés. Dans ces dynamiques, l'univers des messages médiatiques joue ici un rôle crucial.

Le rapport qu'une communauté entretient avec les médias répond à une logique démographique, politique et économique. Une population ne peut disposer de médias que si le nombre de ses citoyens le justifie et elle le fait dans la mesure de cette justification : une petite communauté locale peut bien trouver des ressources pour produire un hebdomadaire, mais elle ne se dotera pas d'un contenu télévisuel soutenu. Des médias s'adresseront d'autant plus probablement à elle qu'elle favorise des bénéfices économiques. Les limites de cette probabilité peuvent être surmontées par des gestes politiques si la communauté a quelque incidence sur le politique. Les dynamiques intérieures à la nation sont ainsi hautement déterminantes. Mais ces dynamiques mêmes subiront aussi des influences extérieures, d'ordre politique, économique ou culturel. Un gouvernement ne peut pas choisir d'opprimer une minorité sans s'exposer à quelques conséquences de la part d'autres gouvernements. Des capitaux extra-communautaires peuvent être injectés au sein de la communauté. L'importance d'une culture donnée au sein d'une population dépend souvent de ses liens avec d'autres cultures. 
Figure 4

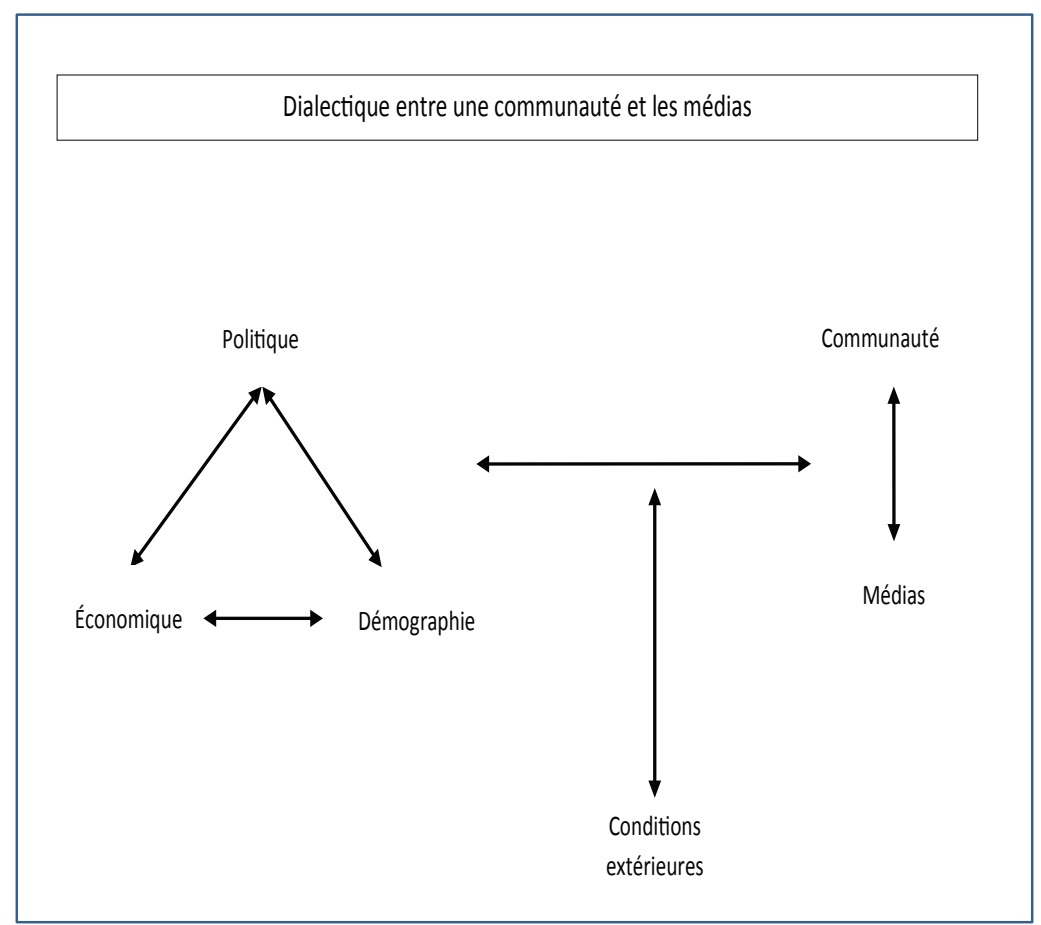

\section{Médias et minorité francophone au Canada}

La francophonie, au Canada, est minorisée par l'anglophonie, c'est-à-dire par le plus puissant producteur de messages de masse.

Dans certains milieux, au pays, les francophones sont suffisamment nombreux pour justifier une logique économique de diffusion de messages de masse : au Québec, certes, mais aussi dans des régions du Nouveau-Brunswick et e l'Ontario (voir Tableau 1). Dans d'autres, ce n'est pas le cas : en Alberta, en Colombie-Britannique, notamment. Au plan national et dans certains environnements provinciaux, les interventions politiques rendent possibles l'accès à des messages médiatiques étendus. Dans certains milieux, la volonté intérieure, démographiquement justifiable, fait en sorte que la logique économique soit marginalisée; des médias diffusent alors dans un esprit coopératif. 


\section{Tableau $1^{4}$}

\begin{tabular}{|c|c|c|c|c|c|c|c|c|c|c|c|c|c|}
\hline \multicolumn{14}{|c|}{$\begin{array}{l}\text { Répartition des médias par province ou territoire } \\
\text { selon la population, sa distribution } \\
\text { et le rapport aux médias de langue majoritaire }\end{array}$} \\
\hline & \multirow{3}{*}{ PROV } & \multirow{3}{*}{$\begin{array}{c}\text { FR } \\
\text { parlé } \\
\text { maison } \\
\%\end{array}$} & \multirow{3}{*}{$\begin{array}{l}\text { En } \\
\text { milliers } \\
\mathrm{N}\end{array}$} & \multirow{2}{*}{\multicolumn{2}{|c|}{ RADIO }} & \multirow{2}{*}{\multicolumn{2}{|c|}{ TÉLÉVISION }} & \multicolumn{4}{|c|}{ JOURNAUX } & \multirow{2}{*}{\multicolumn{2}{|c|}{ TOTAL }} \\
\hline & & & & & & & & QU & DIENS & $\mathrm{HEI}$ & OS & & \\
\hline & & & & FR & $\mathrm{AN}$ & FR & AN & FR & $\mathrm{AN}$ & FR & $\mathrm{AN}$ & MINO & MAJO \\
\hline \multirow{6}{*}{ 矛 } & TNL & 0,1 & $<\mathrm{M}$ & 1 & 24 & 0 & 3 & 0 & 2 & 1 & 1 & 1 & 30 \\
\hline & $\mathrm{NE}$ & 2,0 & $18 \mathrm{M}$ & 2 & 29 & 0 & 5 & 0 & 8 & 1 & 4 & 2 & 46 \\
\hline & NB & 29,7 & $214 \mathrm{M}$ & 11 & 30 & 1 & 6 & 1 & 3 & 5 & 11 & 18 & 43 \\
\hline & IPE & 2,1 & $3 \mathrm{M}$ & 0 & 5 & 0 & 1 & 0 & 2 & 1 & 2 & 1 & 10 \\
\hline & QC & 81,8 & $135 \mathrm{M}$ & 123 & 14 & 36 & 3 & 11 & 6 & 87 & 3 & 26 & 257 \\
\hline & $\mathrm{ON}$ & 2,5 & $305 \mathrm{M}$ & 11 & 182 & 3 & 34 & 1 & 28 & 12 & 64 & 27 & 308 \\
\hline \multirow{4}{*}{ 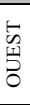 } & MA & 1,8 & $20 \mathrm{M}$ & 2 & 34 & 1 & 5 & 0 & 6 & 1 & 25 & 4 & 70 \\
\hline & SK & 0,5 & $4 \mathrm{M}$ & 1 & 37 & 1 & 7 & 0 & 4 & 1 & 78 & 3 & 126 \\
\hline & ALB & 0,7 & $21 \mathrm{M}$ & 2 & 72 & 1 & 15 & 0 & 15 & 2 & 112 & 5 & 214 \\
\hline & CB & 0,4 & $17 \mathrm{M}$ & 1 & 96 & 1 & 17 & 0 & 32 & 1 & 100 & 3 & 245 \\
\hline \multirow{3}{*}{$\begin{array}{l}\text { वิ } \\
\text { ○ } \\
\text { Z }\end{array}$} & TNO & 1,9 & $<\mathrm{M}$ & 1 & 4 & 0 & 1 & 0 & 0 & 1 & 5 & 2 & 10 \\
\hline & YUK & 1,1 & $<\mathrm{M}$ & 0 & 4 & 0 & 0 & 0 & 1 & 1 & 2 & 1 & 7 \\
\hline & NUN & 0,8 & $<\mathrm{M}$ & 1 & 1 & 0 & 0 & 0 & 0 & 0 & 3 & 1 & 4 \\
\hline \multicolumn{14}{|c|}{$\begin{array}{l}\text { Sources : Annuaire des médias/Media Directory, CCN Matthews News Distribution Experts, Toronto, vol. } 50, \mathrm{n}^{\circ} 2 \text {, } \\
\text { septembre } 2005 \text {; Franco Voyageur, http://francovoyageur.ca, consulté le } 15 \text { décembre } 2009 \text {; Newspapers in Canada, } \\
\text { www.allstuff.com/newsqc.htm, consulté le } 15 \text { décembre } 2009 .\end{array}$} \\
\hline
\end{tabular}

La francophonie canadienne dispose donc de médias, mais dans une moindre proportion que l'anglophonie. Ses médias entrent en concurrence avec un puissant diffuseur dont la puissance est accrue par l'importance de l'anglophonie mondiale. Elle est cependant atténuée par l'importance de la francophonie mondiale. Dans la concurrence internationale des messages médiatiques, l'anglais occupe un immense espace; mais la place du français n'est pas négligeable.

Les messages de l'anglophonie sont accessibles aux francophones du Canada non seulement pour des raisons de démographie ou à cause de l'envergure de la production de messages de langue anglaise, mais aussi par le fait que le minoritaire comprend communément la langue du majoritaire et, de surcroît, à cause de la place symbolique que le majoritaire occupe dans la culture du minoritaire, ce qui exerce une force d'attraction appréciable.

$4 \quad$ Le tableau est tiré de l'article de Christiane Bernier, Simon Laflamme et Sylvie Lafrenière, «L'Effet de la disponibilité des médias et la densité de population minoritaire sur la langue d'usage des médias ", Minorités linguistiques et société, $\mathrm{n}^{\circ} 4$, automne 2013, à paraître. 


\section{Minorité et identité}

Dans ce contexte, le minoritaire peine parfois à se construire une identité qui soit la reproduction de sa caractéristique. Des travaux, comme ceux de Christiane Dallaire et Claude Denis ${ }^{5}$, de Kenneth Deveau, Rodrigue Landry et Réal Allard ${ }^{6}$, Diane GérinLajoie $^{7}$, notamment, montrent à quel point cette identité peut sembler hybride, faite de soi et de l'autre. Depuis longtemps, bon nombre de jeunes francophones en milieu minoritaire au Canada se perçoivent davantage comme bilingues qu'en tant que francophones, ou Canadiens français, ou franco-quelque chose.

Mais l'identité, si elle peut se présenter comme hybride au plan linguistique, elle peut aussi apparaître comme plurielle.

Si l'on propose à des individus des énoncés du type " je m'identifie à... » et qu'on les invite à choisir une valeur sur une échelle à six niveaux allant de " pas du tout à d'accord " à " tout à fait d'accord ", on remarque que les marqueurs sont nombreux et qu'ils ne sont pas mutuellement exclusifs. C'est ce qu'a révélé une enquête menée dans le Nord-Ouest de l'Ontario auprès d'un échantillon d'élèves d'écoles francophones (de la $6^{\mathrm{e}}$ à la $12^{\mathrm{e}}$ année) et d'adultes repérés le plus souvent dans l'environnement des élèves. Nous signalons au passage que si l'on compare entre les élèves et les non-élèves, la plupart de ceux-ci étant des adultes, l'identité est normalement un peu plus définie chez ces derniers (voir Tableau 2) ${ }^{8}$.

5 Christine Dallaire, "Not just Francophone: the Hybridity of Minority Francophone Youths in Canada ", International Journal of Canadian Studies, $\mathrm{n}^{\circ}$ 28, 2003, p. 163-199; Christine Dallaire et Claude Denis, "Asymmetrical Hybridities: Youths at Francophone Games in Canada ", Canadian Journal of Sociology, vol. 30, n 2, 2005, p. 143-168.

6 Kenneth Deveau et Rodrigue Landry, "Identité bilingue : produit d'un déterminisme social ou résultat d'une construction autodéterminée? ", dans Michel Bock, (dir.), La Jeunesse au canada français : formation, mouvements et identité, Ottawa, Presses de l'Université d'Ottawa, 2007, p. 113-134; Rodrigue Landry, Kenneth Deveau et Réal Allard, "Vitalité ethnolinguistique et construction identitaire : le cas de l'identité bilingue ", Éducation et francophonie, vol. 34, $\mathrm{n}^{\circ} 1,2006$, p. 54-81.

7 Diane Gérin-Lajoie, "La Problématique identitaire et l'école de langue française en Ontario ", Francophonies d'Amérique, no 18, 2004, p. 171-179.

8 Nicole Corbett, Simon Laflamme et Chris Southcott, "Usage des médias et 
Les analyses montrent qu'on s'identifie à sa famille, au Canada et à son sexe avant de s'identifier à la francophonie, mais qu'on s'identifie fortement aux quatre. Les moyennes, chez les nonélèves, oscillent entre 4,76 et 5,55 et, chez les jeunes, entre 3,73 et 4,94. Elles sont, dans l'ensemble, bien au-dessus de 4, à proximité ou au-dessus de 5. La plus faible est de 3,73; on la trouve chez les élèves; elle se rapporte à la francophonie; elle est de 1,02 inférieure à celle des non-élèves. Les analyses montrent que ces inclinations prononcées n'empêchent pas qu'on puisse aussi s'identifier par ailleurs à l'Ontario $\left(\bar{x}_{\text {non-élèves }}=4,56 ; \bar{x}_{\text {élèves }}\right.$ $=4,35)$, à l'Ontario français $\left(\bar{x}_{\text {non-élèves }}=4,47 ; \bar{x}_{\text {élèves }}=3,78\right)$, à la francophonie du Nord-Ouest $\left(\bar{x}_{\text {non-élèves }}=4,25 ; \bar{x}_{\text {élèves }}=3,60\right)$, et puis à son métier $\left(\bar{x}_{\text {non-élèves }}=4,77 ; \bar{x}_{\text {élèves }}=3,14\right)$, et puis à une religion $\left(\bar{x}_{\text {non-élèves }}=3,90 ; \bar{x}_{\text {elèves }}=3,60\right)$, et puis à sa communauté de résidence $\left(\bar{x}_{\text {non-élèves }}=3,89 ; \bar{x}_{\text {elèves }}=4,07\right)$, et puis à une équipe sportive $\left(\bar{x}_{\text {non-élèves }}=2,46 ; \bar{x}_{\text {élèves }}=4,01\right)$. On notera les différences entre les adultes et les jeunes sur le thème de la francophonie, les moyennes étant un peu plus faible chez les seconds. Les écarts types sont toujours supérieurs à 1 mais ils n'atteignent jamais 2 . Ils témoignent donc de différences entre les individus sur chacune des thématiques en même temps que d'une certaine entente. 


\section{Tableau 2}

\begin{tabular}{|c|c|c|c|c|c|c|}
\hline \multicolumn{7}{|c|}{$\begin{array}{c}\text { Différence de moyennes pour les objets d'identification } \\
\text { selon qu'on est élève ou non } \\
(1=\text { Pas du tout d'accord ; } 6 \text { = Tout à fait d'accord })\end{array}$} \\
\hline \multirow[t]{2}{*}{ Énoncé } & & \multicolumn{2}{|c|}{ Statut } & \multirow[t]{2}{*}{$\mathrm{t}$} & \multirow[t]{2}{*}{$\mathrm{D}$} & \multirow[t]{2}{*}{$\mathrm{p}<0,0$} \\
\hline & & Non-élève & Élève & & & \\
\hline \multirow{2}{*}{ Je m'identifie à ma famille } & $\bar{x}$ & 5,55 & 4,94 & \multirow{2}{*}{6,29} & \multirow[b]{2}{*}{607,3} & \multirow[b]{2}{*}{ oui } \\
\hline & $\mathrm{s}$ & 1,01 & 1,43 & & & \\
\hline \multirow{2}{*}{ Je m'identifie à la francophonie } & $\bar{x}$ & 4,76 & 3,73 & \multirow{2}{*}{8,23} & \multirow{2}{*}{653} & \multirow{2}{*}{ oui } \\
\hline & $\mathrm{s}$ & 1,57 & 1,63 & & & \\
\hline \multirow{2}{*}{ Je m'identifie à l'Ontario français } & $\bar{x}$ & 4,47 & 3,78 & \multirow{2}{*}{5,35} & \multirow{2}{*}{660} & \multirow{2}{*}{ oui } \\
\hline & $\mathrm{s}$ & 1,64 & 1,67 & & & \\
\hline \multirow{2}{*}{$\begin{array}{l}\text { Je m'identifie à la francophonie du Nord- } \\
\text { Ouest de l'Ontario }\end{array}$} & $\bar{x}$ & 4,25 & 3,60 & \multirow{2}{*}{4,80} & \multirow{2}{*}{653} & \multirow{2}{*}{ oui } \\
\hline & $\mathrm{s}$ & 1,70 & 1,77 & & & \\
\hline \multirow{2}{*}{ Je m'identifie au Nord-Ouest de l'Ontario } & $\bar{x}$ & 4,29 & 3,82 & \multirow{2}{*}{3,43} & \multirow{2}{*}{635} & \multirow[b]{2}{*}{ oui } \\
\hline & $\mathrm{s}$ & 1,69 & 1,76 & & & \\
\hline \multirow{2}{*}{ Je m'identifie à l'Ontario } & $\bar{x}$ & 4,56 & 4,35 & \multirow{2}{*}{1,68} & & \\
\hline & $\mathrm{s}$ & 1,59 & 1,64 & & 636 & non \\
\hline & $\bar{x}$ & 2,95 & 2,89 & & 620 & \\
\hline Je m identifle au Quebec & $\mathrm{s}$ & 1,94 & 1,87 & 0,45 & 629 & non \\
\hline Je m'identifie au Canada & $\bar{x}$ & 5,08 & 4,73 & 200 & 6257 & \\
\hline Je m identinie au Canada & $\mathrm{s}$ & 1,41 & 1,62 & 2,90 & 635,1 & oul \\
\hline & $\bar{x}$ & 4,77 & 3,14 & & & \\
\hline Je m' identitie au metter que j' exerce & $\mathrm{s}$ & 1,58 & 1,82 & 11,83 & 598,8 & oul \\
\hline Je m'identifie à l'organisme pour lequel je & $\bar{x}$ & 4,59 & 3,14 & & & \\
\hline travaille & $\mathrm{s}$ & 1,58 & 1,75 & 10,10 & 514,5 & oul \\
\hline Je m'identifie à mon sexe (au fait d'être & $\bar{x}$ & 4,98 & 4,71 & & & \\
\hline homme ou femme) & $\mathrm{s}$ & 1,53 & 1,73 & 2,04 & 634,6 & oui \\
\hline & $\bar{x}$ & 3,90 & 3,60 & 205 & 619 & oui \\
\hline Je m identifie a ma religion & $\mathrm{s}$ & 1,76 & 1,83 & 2,05 & 619 & oul \\
\hline Je m’identifie à une c cuse nolitioue & $\bar{x}$ & 2,57 & 2,35 & 168 & 638 & \\
\hline Je m identifle a une cause politique & $\mathrm{s}$ & 1,63 & 1,61 & 1,68 & 658 & non \\
\hline & $\bar{x}$ & 3,35 & 3,05 & & & \\
\hline Je m' identitie a une cause sociale & $\mathrm{s}$ & 1,73 & 1,83 & 2,15 & 645 & oul \\
\hline Je m’identifie à ma ville ou à mon village & $\bar{x}$ & 3,89 & 4,07 & $-1,32$ & 641.5 & \\
\hline Je m'identitie a ma ville ou a mon village & $\mathrm{s}$ & 1,62 & 1,81 & $-1,32$ & 641,5 & non \\
\hline intifie ò une & $\bar{x}$ & 2,46 & 4,01 & 1061 & 6380 & ij \\
\hline Je m raentifie a une equipe sportive & $\mathrm{s}$ & 1,75 & 1,96 & $-10,61$ & 038,9 & oul \\
\hline
\end{tabular}

Un francophone minoritaire, n'est pas qu'un francophone minoritaire. L'anglicité de son environnement agit sur lui et en lui. Mais chez lui comme chez bien d'autres citoyens, qui peuvent être majoritaires, il y a d'autres zones d'identification, partageables ou non. Et la multiplicité des sources d'information associée à la postmodernité ainsi que la complexité du rapport à 
l'information d'une société qui permet aussi bien le partage de messages de masse que l'individuation des relations aux médias favorisent ces complexes où l'identité procède de la pluralité aussi bien que de l'uniformité.

L'enjeu linguistique n'est pas le même pour le minoritaire que pour le majoritaire, bien sûr. Le passage vers l'autre est plus communément au détriment de la francophonie. L'hybridité identitaire linguistique est propre beaucoup plus au minoritaire qu'au majoritaire. Le pouvoir d'attraction de la francophonie, au Canada, n'est pas tel qu'il menace démographiquement l'anglophonie. Il n'est pas tel non plus qu'il ne menace pas la francophonie quand elle se tourne vers l'anglophonie. En ce sens, la question de la reproduction est vitale pour la francophonie, et c'est la raison pour laquelle elle fait partie de la culture - et qu'elle est régulièrement posée par les spécialistes. Mais cette culture ne peut pas être que ce questionnement, sinon elle ne pourrait constituer un milieu de partage de symboliques dans un environnement postmoderne. C'est pourquoi il est important pour elle que ses pratiques artistiques et ses pratiques médiatiques la dotent des symboliques qui fassent que son univers se plaise à se différencier.

L'humain peut s'identifier à plusieurs choses. Dans la postmodernité, il le fait aisément. On peut dire en ce sens que les cultures sont plurielles, comme l'est l'identité. Mais quand un définisseur communautaire rebute des membres qui sont issus de l'ensemble, alors, et dans la mesure de cet éloignement, c'est toute la culture qui se transforme. La question est alors de savoir si l'évolution est celle de l'homogénéité ou celle de l'hybridité. Mais l'hybridité n'est possible que sur fond d'homogénéité. En fait, il est d'autant plus probable qu'il y ait hybridité que la culture dont on est issu et qui ne suffit plus continue d'exercer une forte attraction. Et la culture ne peut le faire que si elle constitue un milieu nettement différenciable, donc un milieu auquel un grand nombre s'identifient clairement parce qu'il est animé par des pratiques artistiques et médiatiques enrichissantes avec lesquelles il est possible d'être en correspondance. Si tel n'est 
pas le cas, il ne peut y avoir de mouvement continu vers l'hybridité; il ne peut y avoir que transfert. En fait, l'hybridité du francophone ne pourra durer que dans la mesure où elle coexistera avec une francophonie distincte et qui s'affirme, qui s'affirme moins par les slogans que par le plaisir de l'être, par sa créativité dans la concurrence des créations, par ses usages médiatiques dans la concurrence des médias.

\section{Conclusion}

Une culture ne peut pas être que pluralité parce qu'elle ne peut, alors, réunir ses membres en fonction d'un dénominateur commun. Elle ne peut pas non plus être qu'unité, car elle ne permet pas à ses membres d'être en correspondance avec la particularité de leur histoire. Une communauté vit d'une tension entre une tendance à l'individuation et à une autre à l'homogénéisation.

Culture, identité, francophonie minoritaire, pratique artistiques et pratique médiatiques constituent un tout en ce sens que, pour qu'il y ait francophonie minoritaire, les quatre autres dimensions doivent intervenir.

Pour qu'il y ait culture, il faut qu'il y ait partage de symboles, entre l'émotion et la raison. Dans la postmodernité, le partage de symboles, pour une communauté étendue, n'est possible que s'il implique des moyens de communication de masse, ceux qui destinent des messages à plusieurs et ceux grâce auxquels des masses d'individus peuvent entretenir des relations interpersonnelles en dépit de la multiplicité des lieux.

Le statut de minoritaire est relatif. Il dépend de facteurs intérieurs et extérieurs à l'ensemble au sein duquel évolue la minorité. Mais comme toute communauté, dès lors qu'elle est étendue, celle qui est minoritaire demande une action des moyens de communication de masse. Ces médias ne sont pas que des diffuseurs qui transmettent des messages à la communauté indépendamment de son éthos. Si tel est le cas, elle ne peut se reconnaitre, elle ne peut donc se reproduire, elle ne peut non plus se produire pour les autres. Une communauté postmoderne appelle une action des médias; mais cette action doit procéder 
de l'aptitude de la communauté elle-même à produire des créateurs qui la reproduisent et l'inventent en continu de telle manière que, en lien avec eux, ses membres puissent construire leur être collectif et individuel grâce aux messages qui viennent à eux et qu'ils émettent, immédiatement ou médiatement, et qu'ils puissent le faire dans un contexte de concurrence des pratiques artistiques et des diffusions médiatiques.

\section{Bibliographie}

Bernier, Christiane, Simon Laflamme et Sylvie Lafrenière, "L'Effet de la disponibilité des médias et la densité de population minoritaire sur la langue d'usage des médias ", Minorités linguistiques et société, $\mathrm{n}^{\circ} 4$, automne 2013, à paraître.

Corbett, Nicole, Simon Laflamme et Chris Southcott, «Usage des médias et langue de communication dans la communauté francophone du nord-ouest de l'Ontario ", Revue du Nouvel-Ontario, no 33, 2008, p. 69-94.

Dallaire, Christine, " Not just Francophone: the Hybridity of Minority Francophone Youths in Canada ", International Journal of Canadian Studies, n ${ }^{\circ}$ 28, 2003, p. 163-199.

Dallaire, Christine et Claude Denis, "Asymmetrical Hybridities: Youths at Francophone Games in Canada ", Canadian Journal of Sociology, vol. $30, n^{\circ} 2,2005$, p. 143-168.

Deveau, Kenneth et Rodrigue Landry, "Identité bilingue : produit d'un déterminisme social ou résultat d'une construction autodéterminée?", dans Michel Bock, (dir.), La Jeunesse au canada français : formation, mouvements et identité, Ottawa, Presses de l'Université d'Ottawa, 2007, p. 113-134.

Gérin-Lajoie, Diane, "La Problématique identitaire et l'école de langue française en Ontario ", Francophonies d'Amérique, n 18, 2004, p. 171179.

Landry, Rodrigue, Kenneth Deveau et Réal Allard, «Vitalité ethnolinguistique et construction identitaire : le cas de l'identité bilingue ", Éducation et francophonie, vol. 34, no 1, 2006, p. 54-81. 
Luhmann, Niklas, Systèmes sociaux. Esquisse d'une théorie générale, traduit de l'allemand par Lukas K. Sosoe, Québec, Presses de l'Université Laval, [1984] 2010, 569 p.

McLuhan, Marshall et Quentin Fiore, The Medium is the Message: an Inventory of Effects, New York, Bantam Books et Random House, 1967.

Morin, Edgar, La Méthode, tome 3, La Connaissance de la Connaissance. Anthropologie de la connaissance, Paris, Seuil, 1992. 